\title{
Estudo comparativo da composição química das espécies vegetais Mikania glomerata Sprengel e Mikania laevigata Schultz Bip. ex Baker
}

\author{
Ricardo C. Bolina, ${ }^{1,2}$ Eliana de F. Garcia, ${ }^{2}$ Maria Gorette R. Duarte ${ }^{\star, 1}$ \\ ${ }^{1}$ Divisão de Ciências Farmacêuticas, Fundação Ezequiel Dias, Rua Conde Pereira Carneiro, 80, Gameleira, \\ 30510-010 Belo Horizonte-MG, Brasil, \\ ${ }^{2}$ Centro Universitário Newton Paiva, Av. Silva Lobo, 1720, Nova Granada, 30460-000 Belo Horizonte-MG, Brasil
}

\begin{abstract}
RESUMO: Adulterações da matéria-prima vegetal ocorrem comumente no mercado de fitoterápicos, sob a forma de substituições e/ou falsificações. Muitas vezes, espécies distintas são comercializadas em substituição à farmacopéica devido à dificuldade de obtenção desta, ou mesmo pelo emprego intencional de espécies de menor valor econômico que apresentem características morfológicas semelhantes. Portanto, faz-se necessária a realização de estudos que busquem identificar a constituição química das espécies de amplo uso medicinal, que são alvos de adulterações. Mikania glomerata e Mikania laevigata, conhecidas popularmente como "guaco", são comercializadas indistintamente. Buscando-se avaliar a composição química das duas espécies, realizou-se uma prospecção fitoquímica por $\mathrm{CCD}$ e foram obtidos os perfis cromatográficos por CLAE-FR. Fez-se a quantificação da cumarina empregando-se o mesmo método. Na prospecção fitoquímica por $\mathrm{CCD}$, foi detectada, nas duas espécies, a presença de cumarina, triterpenos/ esteróides e heterosídeos flavônicos. Os perfis cromatográficos, obtidos por CLAE-FR, mostraramse semelhantes, observando-se a predominância de compostos polares. Na quantificação da cumarina, obtêve-se um teor de $0,30 \%$ para $M$. glomerata e $0,43 \%$ para M. laevigata, teores de acordo com o preconizado na monografia do guaco-cheiroso (mín. 0,1\%). M glomerata e $M$. laevigata apresentaram composição química semelhante e teores de cumarina próximos, sugerindo que estas podem ser utilizadas de forma indistinta.
\end{abstract}

Unitermos: Mikania glomerata, Mikania laevigata, Asteraceae, guaco, cumarina.

\begin{abstract}
Comparative study of the chemical composition of the species Mikania glomerata Sprengel and Mikania laevigata Schultz Bip. ex Baker". Adulterations of plant raw materials often occur in the marketing of herbal medicine, usually in the form of substitutions and/ or fakes. Many times, different plant species are commercialized in the place of the one recognized by the pharmacopoeia. This may occur due to the difficulties of obtaining the authentic plant material, or by an intentional use of a plant species that has less economic value but shows similar morphologic features. Therefore, it is necessary to carry out studies that can identify the chemical composition of those species of wide medicinal use, which are the targets of adulterations. Mikania glomerata and Mikania laevigata, popularly known as "guaco", are indistinctly commercialized in Brazil. A phytochemical screening by TLC was carried out to evaluate the chemical composition of both species. The RP-HPLC profiles were obtained and the quantification of coumarin was determined by the same method. A phytochemical screening has shown that coumarin, triterpenes/ steroids and flavonic heterosides are the constituents detected in both species. The RP-HPLC profiles were similar between the two species and revealed the predominance of polar compounds. In the quantification of coumarin, a content of $0.30 \%(\mathrm{w} / \mathrm{w})$ was obtained for $M$. glomerata and $0.43 \%(\mathrm{w} / \mathrm{w})$ for $M$. laevigata. These values are in accord with the contents (min. $0.1 \%$, w/w) described by the monograph of "guaco-cheiroso" in the Brazilian Pharmacopoeia. Therefore, the results indicated similar chemical profiles for M. glomerata and M. laevigata, as well as comparable coumarin contents, thus suggesting that the species might be used indistinctly.
\end{abstract}

Keywords: Mikania glomerata, Mikania laevigata, Asteraceae, guaco, coumarin.

\section{INTRODUÇÃO}

No Brasil, várias espécies nativas têm sido largamente empregadas pela população com fins medicinais, entre estas podem ser citadas espécies de Baccharis (carqueja), Bauhinia (pata-de-vaca), Cecropia (embaúba), Maytenus (espinheira-santa), Mikania (guaco) e Passiflora (maracujazeiro) (Reis et al., 2004; 
Oliveira et al., 2007; Agra et al., 2007, 2008; Corrêa et al., 2008; Marliére et al., 2008; Sousa et al., 2008; Veiga-Junior, 2008). Em relação ao gênero Mikania, as espécies mais utilizadas são Mikania glomerata Sprengel e Mikania laevigata Shultz Bip. ex Baker, conhecidas popularmente como guaco. M. glomerata consta da $1^{\text {a }}$ edição da farmacopéia brasileira e da $\mathrm{RE} \mathrm{n}^{\circ} 89$, que se refere à lista de registro simplificado de fitoterápicos (Pharmacopéia dos Estados Unidos do Brasil, 1926; Brasil, 2004; Brandão et al., 2006, 2008; Carvalho et al., 2008; Rocha et al., 2008), e M. laevigata encontrase descrita no sexto fascículo da farmacopéia brasileira $4^{\mathrm{a}}$ edição (Farmacopéia Brasileira, 2005; Brandão et al., 2006, 2008). A cumarina (1,2-benzopirona) é o marcador químico descrito para as duas espécies (Brasil, 2004; Farmacopéia Brasileira, 2005; Silva et al., 2008).

As espécies $M$. glomerata e $M$. laevigata, usualmente empregadas para o tratamento de distúrbios respiratórios, apresentam semelhanças morfoanatômicas, sendo comercializadas indistintamente. De acordo com trabalhos descritos na literatura, espécies pertencentes a um mesmo gênero podem apresentar diferenças na constituição química. No gênero Eucalyptus, por exemplo, as espécies Eucalyptus globulus Labil e Eucalyptus citriodora Hook, conhecidas popularmente como eucalipto, são utilizadas sem distinção. Segundo Matos (2000), essas espécies apresentam constituição química diferente. O óleo essencial de E. globulus possui o eucaliptol, princípio ativo responsável pela ação expectorante e anti-séptica pulmonar. O óleo essencial de E. citriodora é constituído principalmente pelo citronelal, substância responsável pelo aroma de limão típico dessa planta, mas que não possui as propriedades terapêuticas do eucaliptol, embora também apresente ação anti-séptica local.
Dentro deste contexto, o presente trabalho teve como objetivo avaliar qualitativamente a constituição química das espécies $M$. glomerata e M. laevigata, assim como quantificar a cumarina (1,2-benzopirona).

\section{MATERIAL E MÉTODOS}

\section{Material vegetal}

A espécie $M$. glomerata foi coletada na área de cultivo experimental do Departamento de Fitotecnia da Universidade Federal de Viçosa (UFV), Viçosa, MG e uma exsicata foi armazenada no herbário da UFV (VIC 18246). As folhas selecionadas foram lavadas e submetidas à secagem em estufa ventilada a $40{ }^{\circ} \mathrm{C}$. Após a secagem, o material vegetal foi pulverizado em moinho de facas. As folhas da espécie M. laevigata foram coletadas no Centro Pluridisciplinar de Pesquisas Químicas, Biológicas e Agrícolas da Universidade Estadual de Campinas (CPQBA - UNICAMP). Obteve-se o material vegetal seco, que foi pulverizado em moinho de facas.

\section{Preparo dos extratos etanólicos}

Os extratos foram preparados por percolação exaustiva com etanol. O solvente foi evaporado em evaporador rotatório, sob pressão reduzida, a $60{ }^{\circ} \mathrm{C}$. A seguir, os extratos foram pesados e calculados os extrativos.

\section{Prospecção fitoquímica}

Na prospecção fitoquímica por CCD utilizaramse extratos brutos $(1 \mathrm{mg} / \mathrm{mL}$, em MeOH$)$ e cromatoplacas

Tabela 1. Rendimentos em extrativos dos extratos obtidos para M. glomerata e M. laevigata.

\begin{tabular}{|c|c|c|c|}
\hline Espécies vegetais (folhas) & $\begin{array}{c}\text { Massa (g) do material vegetal } \\
\text { seco }\end{array}$ & $\begin{array}{c}\text { Massa ( } \mathrm{g} \text { ) do extrato } \\
\text { etanólico }\end{array}$ & $\begin{array}{c}\text { Extrativos } \\
(\%)\end{array}$ \\
\hline Mikania glomerata & 2100,0 & 322,5 & 15,4 \\
\hline Mikania laevigata & 1980,0 & 416,7 & 21,0 \\
\hline
\end{tabular}

Tabela 2. Prospecção fitoquímica por CCD dos extratos etanólicos de M. glomerata e M. laevigata.

\begin{tabular}{lcc}
\hline Classes de compostos naturais & M. glomerata & M. laevigata \\
\hline Cumarinas & ++ & ++ \\
Heterosídeos antracênicos & - & - \\
Geninas antracênicas & - & - \\
Triterpenos / esteróides & ++ & - \\
Saponinas & - & - \\
Heterosídeos flavônicos & - & - \\
Geninas flavônicas & - & - \\
Alcalóides & - & - \\
Heterosídeos cardiotônicos & - & - \\
Taninos & - & - \\
Polifenóis & & \\
\hline
\end{tabular}

$(++)$ reação forte; $(+)$ reação fraca; (-) reação negativa. 


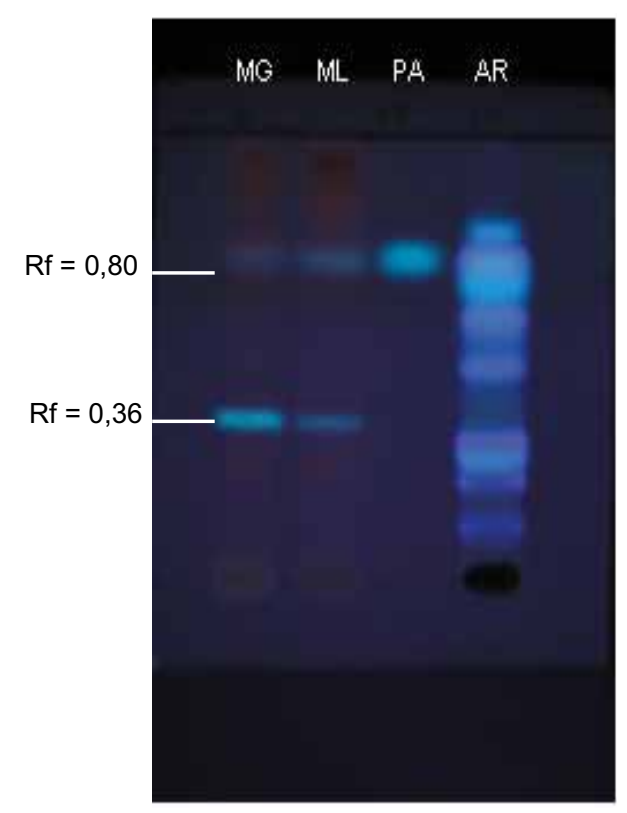

Figura 1. Perfis cromatográficos, obtidos por CCD, para as amostras de $M$. glomerata (MG) e M. laevigata (ML).

PA: cumarina (1,2-benzopirona, Sigma); AR: amostra de referência (Brosimum gaudichaudii - mamacadela) Eluente: tolueno - éter (1:1), saturado com ácido acético a 10\%. Revelador: $\mathrm{KOH}$ a 5\% em etanol, sob luz UV365nm.

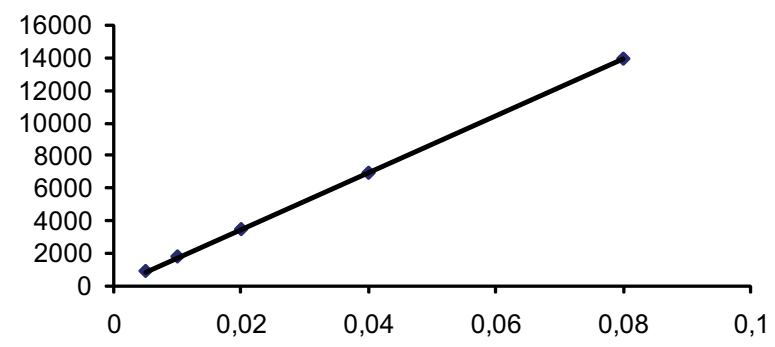

Figura 2. Curva de calibração utilizada na quantificação da cumarina nas folhas de M. glomerata e M. laevigata.

de alumínio recobertas por sílica gel $60 \mathrm{G} \mathrm{F}_{254}$ Merck, visando detectar a presença dos seguintes grupos de metabólitos secundários: cumarinas, geninas e heterosídeos antracênicos, triterpenos / esteróides, saponinas, geninas e heterosídeos flavônicos, alcalóides, glicosídeos cardiotônicos, taninos e polifenóis. Para cada grupo utilizou-se um sistema eluente adequado, reveladores específicos e amostras de referência (Stahl, 1971; Marini-Bettolo et al., 1981; Duarte, 1995; Wagner \& Bladt, 1996; Duarte, 2001).

\section{Determinação de água}

Para a determinação do teor de água utilizouse o método gravimétrico descrito na farmacopéia brasileira (Farmacopéia Brasileira, 2000). As análises foram realizadas em triplicata e os resultados expressos em porcentagem, em relação à droga vegetal. Conforme preconizado pela farmacopéia brasileira, o teor de água contido na amostra é necessário para os cálculos da quantificação de cumarina (Farmacopéia Brasileira, 2005).

\section{Quantificação da cumarina por CLAE-FR}

As análises foram realizadas em cromatógrafo líquido Agilent - mod. HP 1100, equipado com injetor automático, detector UV-VIS, bomba quaternária e software ChemStation. Empregou-se coluna ODS Hypersil $(125 \times 4 \mathrm{~mm}, 5 \mu \mathrm{m})$, fluxo de $0,5 \mathrm{~mL} / \mathrm{min}$, detecção $\mathrm{UV}_{275 \mathrm{~nm}}$ e eluição isocrática $\left(\mathrm{H}_{2} \mathrm{O}-\mathrm{MeOH}\right.$ 53:47) (Aboy et al., 2000). As amostras foram preparadas de acordo com metodologia descrita pela Farmacopéia Brasileira (2005). Posteriormente, as amostras foram centrifugadas (10.000 rpm, por $10 \mathrm{~min}$.) e o sobrenadante foi empregado nas análises $(20 \mu \mathrm{L})$. As amostras foram preparadas em triplicata, fazendo-se três injeções para cada triplicata. A quantificação da cumarina foi feita utilizando-se a cumarina (1,2-benzopirona, Sigma) como padrão externo. Para a construção da curva de calibração preparou-se uma solução estoque a $0,5 \mathrm{mg} / \mathrm{mL} \mathrm{em} \mathrm{MeOH}$ e alíquotas desta foram diluídas em $\mathrm{H}_{2} \mathrm{O}-\mathrm{MeOH}$ (53:47), obtendo-se soluções a 0,$005 ; 0,01 ; 0,02 ; 0,04$ e 0,08 $\mathrm{mg} / \mathrm{mL}$ (Celeghini et al., 2001), que foram injetadas em quintuplicata. Calculou-se o teor da cumarina a partir da equação da reta obtida com a curva de calibração. $\mathrm{O}$ resultado foi expresso pela média das determinações em gramas de cumarina por $100 \mathrm{~g}$ da amostra, considerandose a determinação de água (\%).

\section{Obtenção dos perfis cromatográficos por CLAE-FR}

As análises foram realizadas em cromatógrafo líquido Agilent - mod. HP 1100, equipado com injetor automático, detector UV-VIS, bomba quaternária e software ChemStation. Empregou-se coluna ODS Hypersil $(125 \times 4 \mathrm{~mm}, 5 \mu \mathrm{m})$, fluxo de $1,0 \mathrm{~mL} / \mathrm{min}$, detecção $\mathrm{UV}_{210 \mathrm{~nm}}$ e eluição isocrática $\left(\mathrm{CH}_{3} \mathrm{CN}-\mathrm{H}_{2} \mathrm{O}\right.$ 15:85). O extrato bruto foi solubilizado em $\mathrm{MeOH}$ (10 $\mathrm{mg} / \mathrm{mL}$ ) e centrifugado (10.000 rpm, $10 \mathrm{~min}$.), sendo o sobrenadante utilizado nas análises por CLAE $(20 \mu \mathrm{L})$.

\section{RESULTADOS E DISCUSSÃO}

As massas do material vegetal seco utilizado e dos extratos etanólicos obtidos da percolação, assim como os rendimentos em extrativos constam na Tabela 1. Na prospecção fitoquímica realizada por CCD constatouse a presença da cumarina (1,2-benzopirona), triterpenos/ esteróides e heterosídeos flavônicos no extrato etanólico de M. glomerata e M. laevigata (Tabela 2). A presença de cumarina, triterpenos e esteróides está de acordo com as descrições feitas por Oliveira et al. (1984), Oliveira et al. 

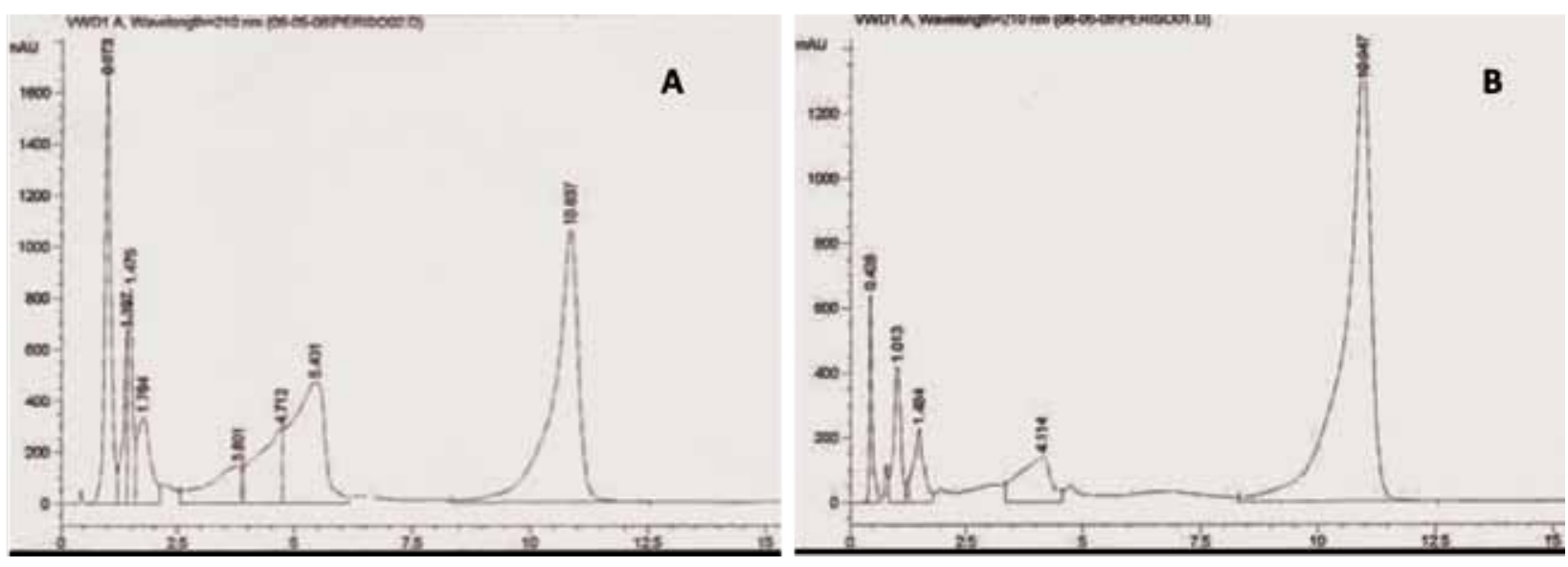

Figura 3. Perfis cromatográficos, obtidos por CLAE-FR, para os extratos etanólicos de M. glomerata (A) e M. laevigata (B). Condições cromatográficas: vide Material e Métodos.

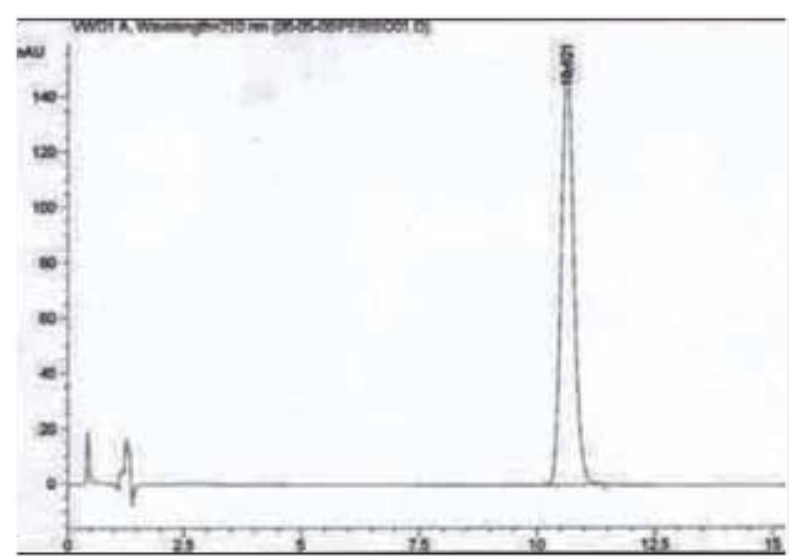

Figura 4. Cromatograma da cumarina padrão $(0,02 \mathrm{mg} / \mathrm{mL})$. Condições cromatográficas: vide Material e Métodos.

(1994), Santos et al. (1999), Amaral et al. (2003), Matos et al. (2004), Pedroso et al. (2008) e Silva et al. (2008). Obtêve-se resultado positivo para heterosídeos flavônicos, embora Oliveira et al. (1984) não tenham constatado a presença desses na espécie. Geninas e heterosídeos antracênicos, saponinas, geninas flavônicas, alcalóides, heterosídeos cardiotônicos, taninos e polifenóis simples não foram detectados em ambas as espécies. Entretanto, em trabalho realizado por Oliveira et al. (1984), foi relatada a presença de alcalóides, saponinas, taninos e polifenóis nas partes aéreas de $M$.glomerata e $M$. laevigata. Na prospecção fitoquímica para detecção de cumarinas, observou-se no perfil cromatográfico das duas espécies, a presença de uma mancha fluorescente esverdeada $(\mathrm{Rf}=0,36)$, provavelmente relacionada ao ácido o-cumárico e uma mancha de fluorescência verde, de $\mathrm{Rf}=0,80$, referente à cumarina (Farmacopéia Brasileira, 2005) (Figura 1).

Considerando que não existem limites farmacopéicos descritos para os teores de água e cumarina nas folhas de $M$. glomerata, utilizaram-se como parâmetros os valores preconizados pela monografia do guaco-cheiroso (M. laevigata). Os teores de água obtidos para M. glomerata $(10,4 \% \pm 0,2)$ e $M$. laevigata $(8,7 \%$ $\pm 0,1$ ), mostraram-se dentro do limite estabelecido pela farmacopéia brasileira, no máximo 10\% (Farmacopéia Brasileira, 2005). Na quantificação da cumarina por CLAE-FR, a equação da reta obtida por regressão linear para a curva padrão foi $y=174557,846 x-17,096$, onde " $y$ " representa a área dos picos e " $x$ " a concentração de cumarina $(\mathrm{mg} / \mathrm{mL})$ (Figura 2). O coeficiente de correlação (r) obtido foi de 0,9999, denotando comportamento linear na faixa de concentração avaliada. As folhas de $M$. glomerata e M. laevigata apresentaram teores de cumarina de $0,30 \% \pm 0,01$ e $0,43 \% \pm 0,02$, respectivamente, estando estes valores acima do exigido pela Farmacopéia Brasileira para o guaco-cheiroso (mín. 0,1\%) (Farmacopéia Brasileira, 2005). A quantificação por CLAE-FR mostrou que a espécie M. laevigata apresentou maior teor de cumarina.

Os perfis cromatográficos por CLAE-FR (Figura 3) foram determinados com a finalidade de se conhecer a composição qualitativa das duas espécies vegetais. As amostras avaliadas apresentaram perfis semelhantes, observando-se predominância de compostos polares, com TR menores que seis min., o que pode ser atribuído ao processo de extração empregado, percolação com etanol. A cumarina apresentou um TR de aproximadamente 10,62 min. (Figura 4), sendo detectada nas duas espécies como componente majoritário, por apresentar um maior valor de área sob o pico em relação aos outros constituintes.

\section{CONCLUSÃO}

Pelos perfis cromatográficos obtidos por $\mathrm{CCD}$, constatou-se que as duas espécies apresentaram constituição química semelhante, sendo detectada a presença da cumarina (1,2-benzopirona), triterpenos/ esteróides e heterosídeos flavônicos. Características semelhantes foram também observadas nos perfis cromatográficos dos extratos etanólicos de $M$. glomerata e M. laevigata obtidos por CLAE-FR. e os teores de cumarina mostraram-se bastante próximos. Assim, 
os resultados encontrados sugerem que as espécies $M$ glomerata e M. laevigata podem ser utilizadas de forma indistinta.

\section{AGRADECIMENTOS}

Ao Prof. Ricardo Henrique Silva Santos (UFV) pelo fornecimento da amostra de M. glomerata e ao pesquisador Pedro Melillo de Magalhães (CPQBA - UNICAMP) pelo fornecimento da amostra de $M$. laevigata.

\section{REFERÊNCIAS}

Aboy AL, Petrovik GOPR, Langeloh VLB 2000. Desenvolvimento de soluções extrativas de Mikania glomerata Sprengel (Asteraceae), guaco. Rev Bras Cienc Farm 36: 165-172.

Agra MF, França PF, Barbosa-Filho JM 2007. Synopsis of the plants known as medicinal and poisonous in Northeast of Brazil. Rev Bras Farmacogn 17: 114-140.

Agra MF, Silva KN, Basílio IJLD, França PF, Barbosa-Filho JM 2008. Survey of medicinal plants used in the region Northeast of Brazil. Rev Bras Farmacogn 18: 472508.

Amaral RR, Arcenio Neto F, Carvalho ES, Teixeira LA, De Araújo GL, Sharapin N, Testa B, Gnerre C, Rocha L 2003. Avaliação da atividade IMAO e antibacteriana de extratos de Mikania glomerata Sprengel. Rev Bras Farmacogn 13: 24-27.

Brandão MGL, Cosenza GP, Moreira RA, Monte-Mor RLM 2006. Medicinal plants and other botanical products from the Brazilian Official Pharmacopoeia. Rev Bras Farmacogn 16: 408-420.

Brandão MGL, Zanetti NNS, Oliveira GRR, Goulart LO, MonteMor RLM 2008. Other medicinal plants and botanical products from the first edition of the Brazilian Official Pharmacopoeia. Rev Bras Farmacogn 18: 127-134.

Brasil 2004. Ministério da Saúde. Agência Nacional de Vigilância Sanitária, Resolução - RE no 89, de 16 de março de 2004. Lista de Registro Simplificado de Fitoterápicos. Brasília.

Carvalho ACB, Balbino EE, Maciel A, Perfeito JPS 2008. Situação do registro de medicamentos fitoterápicos no Brasil. Rev Bras Farmacogn 18: 314-319.

Celeghini RMS, Vilegas JHY, Lanças FM 2001. Extraction and quantitative HPLC analysis of coumarin in hydroalcoholic extratcts of Mikania glomerata Spreng. (guaco) leaves. J Braz Chem Soc 12: 706-709.

Corrêa MFP, Melo GO, Costa SS 2008. Substâncias de origem vegetal potencialmente úteis na terapia da Asma. Rev Bras Farmacogn 18 (Supl.): 785-797.

Duarte MGR 1995. Farmacoquimica de plantas invasoras de uso medicinal. Estudos farmacoquimicos de espécies de Polygonum: P. hydropiperoides Mich., P. spectabile Mart. e P. acuminatum H.B.K. Belo Horizonte, 82p. Monografia de Especialização - Faculdade de Farmácia, Universidade Federal de Minas Gerais.

Duarte MGR 2001. Plantas Invasoras Medicinais: Triagem de espécies dos gêneros Cuphea, Desmodium, Polygonum e Sida para atividade antimicrobiana e estudo fitoquímico biomonitorado de Polygonum spectabile Mart. Belo Horizonte, 195 p. Dissertação de Mestrado - Faculdade de Farmácia, Universidade Federal de Minas Gerais.
Farmacopéia Brasileira 2000. 4. ed. São Paulo: Editora Atheneu, parte II, segundo fascículo.

Farmacopéia Brasileira 2005. 4. ed. São Paulo: Editora Atheneu, parte II, sexto fascículo.

Marini-Bettolo GB, Nicletti M, Patamia M, Galeffi G, Messana I 1981. Plant screening by chemical and chromatographic procedures under field conditions. $J$ Chromatogr 213: 113-127.

Marliére LDP, Ribeiro AQ, Brandão MGL, Klein CH, Acurcio FA 2008. Utilização de fitoterápicos por idosos: resultados de um inquérito domiciliar em Belo Horizonte (MG), Brasil. Rev Bras Farmacogn 18 (Supl.): 754-760.

Matos FJA 2000. Plantas Medicinais: Guia de seleção e emprego de plantas usadas em fitoterapia no nordeste do Brasil. Fortaleza: Editora UFC.

Matos FJA, Craveiro AA, Souza MP, Matos MEO, Machado MIL 2004. Constituintes químicos ativos $e$ propriedades biológicas de plantas medicinais brasileiras. Fortaleza: Editora UFC.

Oliveira F, Alvarenga MA, Akisue MK 1984. Isolamento e identificação de componentes químicos de Mikania glomerata Sprengel e de Mikania laevigata Schultz Bip. Ex Baker. Rev Farm Bioquim Univ São Paulo 20: 169-183.

Oliveira F, Saito ML, Garcia LO 1994. Morfologia externa das partes áreas e anatomia foliar das espécies Brasileiras de Mikania Willdenow secção globosae Robinson visão farmacognóstica. Lecta 12: 23-65.

Oliveira FQ, Gobira B, Guimarães C, Batista J, Barreto M, Souza M 2007. Espécies vegetais indicadas na odontologia. Rev Bras Farmacogn 17: 466-476.

Pedroso APD, Santos SC, Steil AA, Deschamps F, Barison A, Campos F, Biavatti MW 2008. Isolation of syringaldehyde from Mikania laevigata medicinal extract and its influence on the fatty acid profile of mice. Rev Bras Farmacogn 18: 63-69.

Pharmacopéia dos Estados Unidos do Brasil 1926. 1.ed. São Paulo: Companhia Editora Nacional.

Reis S, Mariot A, Steenbock W 2004. Diversidade e domesticação de plantas medicinais. In: Simões CMO et al. Farmacognosia: da planta ao medicamento. 5. ed. Florianópolis/Porto Alegre: UFSC/UFRGS, p.4574.

Rocha L, Lucio EMA, França HS, Sharapin N 2008. Mikania glomerata Spreng: Desenvolvimento de um produto fitoterápico. Rev Bras Farmacogn 18 (Supl.): 744747.

Santos PA, Pereira MAS, França SC, Lopes NP 1999. Esteróides e cumarina em calos de Mikania glomerata Sprengel. Rev Bras Cienc Farm 35: 231-235.

Silva CR, Gomes VS, Kulkamp IC, Kanis LA 2008. Método espectroscópico para determinação de cumarina em xarope de Mikania glomerata Sprengel. Rev Bras Farmacogn 18: 594-599.

Sousa FCF, Melo CTV, Citó MCO, Félix FHC, Vasconcelos SMM, Fonteles MMF, Barbosa-Filho JM, Viana GSB 2008. Plantas medicinais e seus constituintes bioativos: Uma revisão da bioatividade e potenciais benefícios nos distúrbios da ansiedade em modelos animais. Rev Bras Farmacogn 18: 642-654.

Stahl E 1971. Analyse chromatographique et microscopique des drogues. Paris: Tech. Et Doc.

Veiga-Junior VF 2008. Estudo do consumo de plantas medicinais na Região Centro-Norte do Estado do Rio de Janeiro: aceitação pelos profissionais de saúde e modo de uso pela população. Rev Bras Farmacogn 18: 308-313.

Wagner H, Bladt S 1996. Plant drug analysis: a thin layer chromatography atlas. Berlin: Springer. 